\title{
Perspective of the Sound Quality Approach Applied to Noise Control in Earth Moving Machines
}

\author{
Giovanni Brambilla \\ Institute of Acoustics O.M. Corbino (IDAC), National Research Council of Italy, \\ Via del Fosso del Cavaliere 100, I-00133, Rome, Italy \\ Eleonora Carletti ${ }^{\dagger}$ and Francesca Pedrielli \\ CEMOTER Institute, National Research Council of Italy, Via Canal Bianco 28, I-44044 Cassana (FE), Italy
}

(Received 5 January 2000; revised 20 November 2000; accepted 4 February 2001)

\begin{abstract}
The paper describes two experiments aimed at evaluating the feasibility and potential of the "sound quality" approach in improving the design of noise reduction treatments for Earth-Moving Machines (EMMs). In both experiments, binaural noise recordings taken in EMMs operating at stationary idle conditions have been used and subsequent laboratory listening tests have been carried out. In the first experiment, the recordings taken in three EMMs at different engine speeds were considered and presented by headphones to 35 naive subjects using the paired comparison method. In the second experiment, one of the previous recordings has been modified to get six sound stimuli, all having equal overall unweighted $L_{e q}$ levels but with different spectral shapes. The results confirm that subjective preference for a sound stimulus is influenced both by the overall sound energy and by its distribution in the frequency domain. Thus, sound energy-oriented parameters turn out to be not sufficient to characterise the auditory perception in EMMs. To investigate this aspect deeply, a principal component analysis has been performed on the sound stimuli. The results show that the most important factors which explain the subjective preference include the 400-5000 Hz frequency range and low engine order harmonics. In addition, loudness and PSIL appear to be promising for assistance with choosing suitable noise treatments more related to auditory perception.
\end{abstract}

${ }^{\dagger}$ Member of the International Institute of Acoustics and Vibration (IIAV)

\section{INTRODUCTION}

Nowadays the commercial success of Earth-Moving Machines (EMMs) on the market depends to a large extent on proper design methods aimed at improving their performance. Among several factors involved (i.e. efficiency, reliability, fuel consumption and cost), low noise emission is increasing in importance. To some extent, this trend is due to the development in Europe, over the last decade, of a framework legislation aimed at health and environmental protection and at improving safety at the workplace. The European Community Directive on noise emission by $\mathrm{EMMs}^{1}$ has established limits for airborne noise emissions (A-weighted sound power level $L_{W_{A}}$ ). In addition, national legislations have laid down noise limits at the workplace (equivalent continuous Aweighted sound pressure level $\left.L_{A_{e q}}\right)$. Thus, EMM manufacturers have been focused mainly on reducing both $L_{W_{A}}$ and $L_{A_{e q}}$ levels to comply with the above limits and to improve the acoustical climate inside the cabin. The rationale for this approach might be summarised in the statement: "lower is better". ${ }^{2}$

In achieving these objectives, significant noise reductions have been obtained inside EMM cabins at present: noise levels lower than $90 \mathrm{~dB}(\mathrm{~A})$ frequently occur, the values generally being within the range $80-85 \mathrm{~dB}(\mathrm{~A})$.

However, research carried out in recent years has shown that even if noise levels fulfil the limits issued by legislation, they can still elicit negative responses from subjects, such as annoyance and interference with activities, which both decrease work performance. Furthermore, as different noises which have equal values of $L_{A_{e q}}$ can evoke different subjective reactions, ${ }^{3}$ energy-oriented parameters, such as sound power or sound pressure levels, are not always sufficient to describe auditory perception, unless the source is very loud. Hence, even if "safe" noise levels are achieved, other objective noise descriptors more related to auditory perception must be taken into account. Such a more detailed analysis can provide useful hints for improving the acoustical performance of the product.

The sound quality approach has been recognised as a powerful tool to handle such subjective aspects of noise and it is widely applied, especially in the automotive industry where the acoustical comfort in the car is a more and more influencing factor in determining a purchaser's appraisal. As defined by Blauert and Bodden, ${ }^{4}$ sound quality is the adequacy of a sound in the context of a specific technical goal and/or task. The term "compatibility" has also been used instead of "adequacy", especially with regard to sounds accompanying actions of the users of the products. ${ }^{5}$ Such a concept of quality involves two additional aspects: 1) the sound should be pleasant or at least not unpleasant; and 2) it should be identifiable. More generally for industrial products, the concept of "product sound quality" can be defined as "...a descriptor of the adequacy of the sound attached to a product. It results from judgements upon the totality of the auditory 